\title{
CONTROLE DE QUALIDADE EM RADIOGRAFIAS PERIAPICAIS OBTIDAS NO CURSO DE ODONTOLOGIA DA UEPG
}

\author{
CONTROL OF QUALYTY IN PERIAPICAL \\ RADIOGRAPHIES FROM THE DENTISTRY \\ COURSE IN UEPG
}

\author{
ELIANE MARIA KREICH ${ }^{1}$ \\ MÁRCIA G. SCHWEITZER QUEIROZ² \\ MARIANE CRISTINA SLONIAK ${ }^{2}$ \\ 1 Professora do Departamento de Odontologia da \\ UEPG \\ 2 Acadêmica do $5^{\circ}$ ano do Curso de Odontologia \\ da UEPG
}

\begin{abstract}
RESUMO
O exame radiográfico é um meio complementar importante e necessário, que o profissional da área da saúde utiliza na elaboração do diagnóstico, planejamento e execução do tratamento, proservação de casos clínicos e documentação de um modo geral. Para uma interpretação correta e segura, há necessidade de radiografias com padrão de qualidade aceitável, que possibilitem um diagnóstico adequado. Com o objetivo de aprimorar a qualidade da imagem radiográfica, foram analisadas radiografias intrabucais periapicais, realizadas por alunos dos $2^{\circ}, 3^{\circ}, 4^{\circ}$ e $5^{\circ}$ anos do Curso de Odontologia da UEPG, nos anos de
\end{abstract}


1996 e 1997, levando em consideração os critérios de avaliação: técnica, processamento e densidade radiográfica. Os resultados obtidos foram comparados entre os diferentes anos de graduação, e avaliada a capacidade de aperfeiçoamento dos alunos, com a experiência adquirida no decorrer do curso. Constatou-se que $93,1 \%$ das radiografias examinadas apresentaram algum tipo de erro, sendo que os mais freqüentes foram: posicionamento incorreto do filme $(57,0 \%)$, radiografia clara (55,8\%), encurtamento da imagem (24,9\%), alongamento da imagem (20,1\%), angulação horizontal incorreta (18,4\%). Os autores concluíram ainda que os índices de erros apresentaram percentuais semelhantes em todos os anos de graduação, no decorrer do curso.

Palavras-chave: radiografias intrabucais; erros de técnica/processamento; controle de qualidade

\section{Introdução}

Com a descoberta dos raios X por Wilhelm Conrad Roentgen em 1895, a Odontologia teve um grande avanço, pois a radiografia veio a complementar e solucionar dúvidas de diagnóstico que freqüentemente aparecem durante o exame clínico, permitindo ao profissional um planejamento mais seguro do tratamento, além de sua utilização na proservação e documentação de um modo geral. Portanto, este recurso fornece aos profissionais das diversas especialidades odontológicas, informações úteis para o seu dia-a-dia, desde que empregado corretamente.

Falhas na execução da tomada radiográfica ou durante o processamento dos filmes geram interpretações errôneas, repetição da técnica, maior exposição dos pacientes à radiação, além de aumentar o tempo clínico e os custos dispensados.

A baixa qualidade das imagens já foi apontada pela Organização Mundial de Saúde (OMS) como responsável pela redução na certeza de diagnósticos, desacreditando a radiografia como instrumento de diagnóstico (YACOVENCO, 2001).

O controle da qualidade das radiografias é de responsabilidade do cirurgião-dentista e, com o objetivo de analisar as principais causas da perda desta qualidade e a freqüência com que ocorrem, foi desenvolvida esta pesquisa, onde foi verificado o desempenho dos alunos das clínicas dos $2^{\circ}$, $3^{\circ}, 4^{\circ}$ e $5^{\circ}$ anos do Curso de Odontologia da Universidade Estadual de Pon- 
ta Grossa, nos anos de 1996 e 1997, em relação a técnica e ao processamento durante a obtenção da imagem radiográfica. Os resultados foram comparados entre os diferentes anos de graduação e avaliada a capacidade de aperfeiçoamento dos alunos em relação à prática radiográfica, com a experiência adquirida no decorrer do curso.

\section{Material e métodos}

Foram examinadas 800 radiografias de pacientes submetidos a tratamento em clínicas dos $2^{\circ}, 3^{\circ}, 4^{\circ}$ e $5^{\circ}$ anos do Curso de Odontologia da Universidade Estadual de Ponta Grossa, as quais foram realizadas pela técnica periapical da bissetriz e processadas pelo método visual.

Do total de radiografias analisadas, 400 eram do ano de 1996 e a outra metade correspondia ao ano de 1997, sendo todas consideradas aceitáveis para diagnóstico, porém com erros. As radiografias consideradas inaceitáveis foram descartadas.

Os exames radiográficos foram obtidos de prontuários de pacientes em disciplinas dos diferentes anos de graduação $\left(2^{\circ}, 3^{\circ}, 4^{\circ}\right.$ e $\left.5^{\circ}\right)$, e selecionados aleatoriamente. Em cada ano de graduação foi analisado um total de 200 radiografias, sendo 100 em 1996 e 100 em 1997. A Radiologia foi uma das disciplinas selecionadas, a qual é ministrada no $2^{\circ}$ ano do Curso.

A análise da qualidade da imagem radiográfica foi realizada separadamente por ano de graduação, e depois comparados os resultados entre os diferentes anos. Foram considerados os seguintes critérios de avaliação: Técnica, Processamento e Densidade da Radiografia.

Os erros de Técnica considerados no estudo foram:

$\rightarrow$ Enquadramento - subdividido em posicionamento incorreto do filme e aparecimento da imagem do diafragma;

$\rightarrow$ Angulagem - subdividida em angulação vertical (alongamento e encurtamento) e angulação horizontal (superposição das imagens);

$\rightarrow$ Curvatura do filme - filmes excessivamente curvados devido anatomia de determinadas regiões intrabucais, resultando em imagem distorcida;

$\rightarrow$ Picote - radiografias em que o picote não está voltado para a oclusal/ 
36

incisal, dificultando a identificação do quadrante radiografado;

$\rightarrow$ Tremidas - radiografias com contornos não nítidos, devido ao movimento do filme, aparelho ou paciente durante a exposição;

$\rightarrow$ Dupla exposição - radiografias cujo filme foi exposto mais de uma vez;

$\rightarrow$ Filme invertido - radiografias com o filme invertido em relação à sua face sensível, exposto do lado da lamínula de chumbo.

Os erros de Processamento considerados foram:

$\rightarrow$ Lavagem - radiografias amareladas;

$\rightarrow$ Manchas brancas - gotas de fixador em contato com o filme antes do processamento;

$\rightarrow$ Velamento - radiografias embaçadas com falta de contraste e detalhe;

$\rightarrow$ Bolhas de ar - o ar é aprisionado na superfície do filme após este ser colocado na solução processadora, impedindo os produtos químicos de afetarem a emulsão naquela área;

$\rightarrow$ Reticulação do filme - radiografias com ruptura da emulsão devido a repentina mudança de temperatura entre o revelador e a água de banho;

$\rightarrow$ Impressão digital - filmes manuseados com dedos contaminados;

$\rightarrow$ Eletricidade estática - filmes abertos rapidamente, principalmente em períodos de baixa umidade;

$\rightarrow$ Riscadas - radiografias arranhadas, tendo a emulsão removida da base do filme.

Os erros de Densidade considerados foram:

$\rightarrow$ Radiografias claras - subexpostas e/ou subreveladas;

$\rightarrow$ Radiografias escuras - superexpostas e/ou superreveladas.

Foi anotada a quantidade de erros de técnica, de processamento, de densidade, e o total de erros, pois estes foram identificados e descritos isoladamente durante o exame de cada radiografia.

Para a avaliação das imagens radiográficas, utilizou-se negatoscópios em sala escura com o auxílio de lupas, sendo analisadas uma a uma, e um protocolo de pesquisa, onde se anotou as informações anteriormente mencionadas. 


\section{Resultados e discussão}

Dentre as 800 radiografias analisadas, apenas 6,9\%, que corresponde a 55 radiografias, foram consideradas perfeitas, ou seja, não apresentavam nenhum tipo de erro. O restante apresentou 1847 erros, sendo 1160 erros de técnica, 213 erros de processamento e 474 erros de densidade.

A distribuição dos diferentes tipos de erros de técnica, de processamento e de densidade, assim como das radiografias corretas, nos $2^{\circ}, 3^{\circ}, 4^{\circ}$ e $5^{\circ}$ anos de graduação, pode ser analisada nas tabelas 1,2 e 3 . A média percentual dos 4 anos de graduação para cada tipo de erro, pode ser verificada nos gráficos 1 , 2 e 3.

Tabela 1 - Análise dos erros de técnica

\begin{tabular}{l|c|c|c|c}
\hline \multicolumn{5}{c}{ Radiografias dos diferentes anos de graduação } \\
Curso de Odontologia - UEPG - 1996/97 \\
\hline \multicolumn{1}{c|}{ ERROS } & $\begin{array}{c}2^{\circ} \text { ANO } \\
(\%)\end{array}$ & $\begin{array}{c}3^{\circ} \text { ANO } \\
(\%)\end{array}$ & $\begin{array}{c}4^{\circ} \text { ANO } \\
(\%)\end{array}$ & $\begin{array}{c}5^{\circ} \text { ANO } \\
(\%)\end{array}$ \\
\hline 01 - Posicionamento incorreto do filme & 49,0 & 58,0 & 63,0 & 58,0 \\
02 - Imagem do diafragma & 14,5 & 22,0 & 5,0 & 3,0 \\
03 - Alongamento da imagem & 21,0 & 17,0 & 20,5 & 22,0 \\
04 - Encurtamento da imagem & 24,0 & 23,0 & 23,5 & 29,0 \\
05 - Angulação horizontal incorreta & 14,0 & 23,0 & 17,5 & 19,0 \\
06 - Curvatura do filme & 6,5 & 18,0 & 8,5 & 9,0 \\
07 - Posicionamento incorreto do picote & 1,5 & 6,0 & 2,5 & 0,0 \\
08 - Radiografias tremidas & 0,0 & 1,0 & 0,0 & 1,0 \\
09 - Dupla exposição & 0,5 & 0,0 & 0,0 & 0,0 \\
10 - Filme invertido & 0,0 & 0,0 & 0,0 & 0,0 \\
\hline
\end{tabular}


Pode-se observar conforme Tabela 1, que o erro de maior ocorrência relacionado à técnica, foi o posicionamento incorreto do filme, com uma média entre os diferentes anos de graduação de 57,0\% apontado no Gráfico 1 , o que corresponde a 456 radiografias; em seguida têm-se erros relacionados à angulação vertical, sendo encurtamento da imagem com 24,9\% (199 radiografias) e alongamento da imagem com 20,1\% (161 radiografias); na seqüência tem-se o erro de angulação horizontal com uma média de 18,4\% (146 radiografias); imagem do diafragma com 11,1\% (89 radiografias); curvatura do filme com 10,5\% (84 radiografias); posicionamento incorreto do picote com 2,5\% (20 radiografias). Erros como: radiografias tremidas, dupla exposição e filme invertido tiveram baixa incidência, sendo praticamente insignificantes.

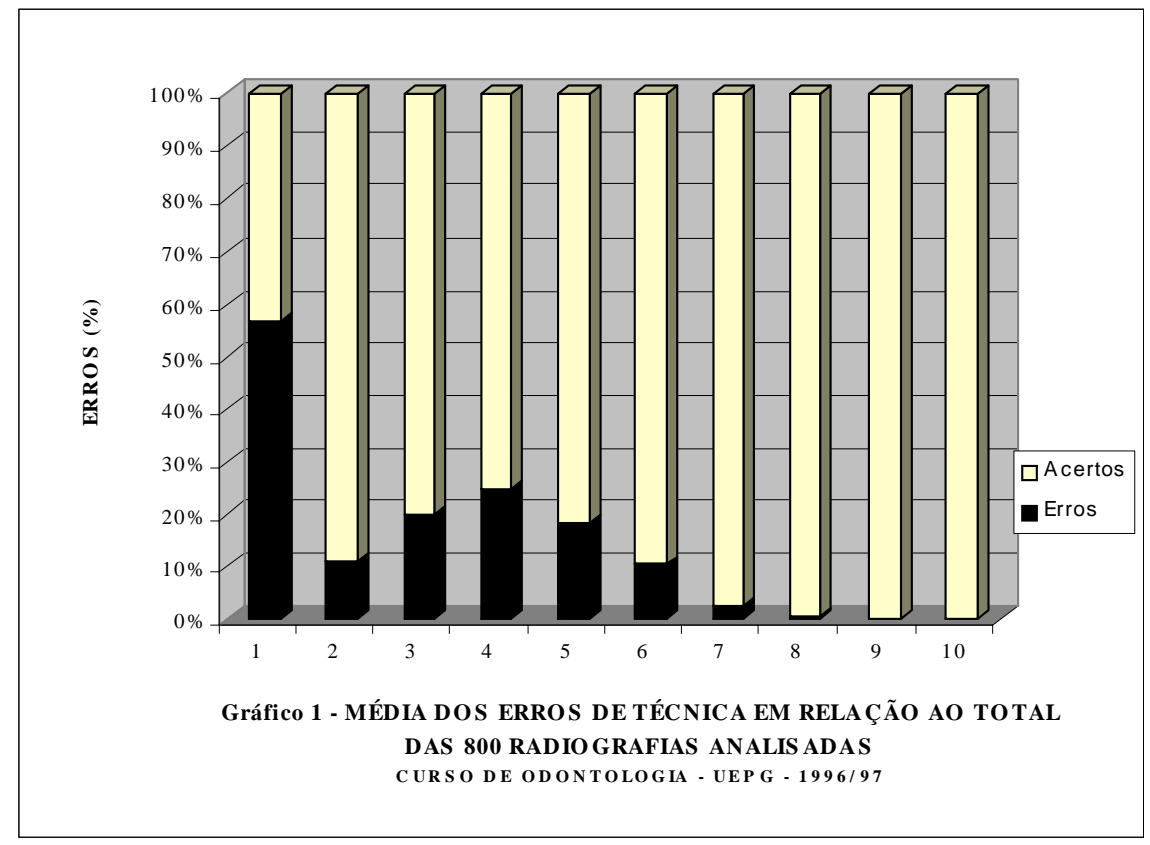

PUBLICATIO UEPG - Ciências Biológicas e da Saúde, 8 (1): 33-45, 2002. 
Trabalhos semelhantes encontrados na literatura mostraram grande número de radiografias com erros. Mourshed (1971), observou também que o erro mais freqüente foi o posicionamento incorreto do filme com $41,8 \%$, seguido da imagem do diafragma (26,1\%), angulação horizontal incorreta (20,6\%) e angulação vertical incorreta (9,1\%).

Patel (1979) concluiu em seu estudo que o posicionamento incorreto do filme foi de longe a maior causa de retomadas radiográficas com $49,9 \%$, seguido pela imagem do diafragma (20,8\%) e angulação vertical incorreta $(12,5 \%)$.

Em outro estudo, Patel e Greer (1986) se propuseram a determinar a presença e os tipos de erros encontrados em levantamentos radiográficos executados por alunos, comparando o aperfeiçoamento da técnica no decorrer do curso. $\mathrm{O}$ erro mais predominante foi o posicionamento incorreto do filme (64,9\%), seguido da angulação vertical incorreta (11,7\%), imagem do diafragma $(11,2 \%)$ e angulação horizontal incorreta $(4,6 \%)$. Os autores concluíram que há a necessidade dos alunos realizarem de 20 a 25 levantamentos periapicais para que possam adquirir uma proficiência técnica mínima.

Gasparini et al. (1992) verificaram que os erros mais freqüentes foram: posicionamento incorreto do filme $(41,4 \%)$, alongamento da imagem (37,6\%) e erro de angulação horizontal (17,48\%).

Avaliando a qualidade das radiografias realizadas na disciplina de Endodontia, Matheus et al. (2000), verificaram que as falhas de maior incidência referem-se à angulação vertical incorreta $(21,0 \%)$ e enquadramento inadequado (19,5\%).

Os resultados do nosso trabalho coincidem com os da literatura, onde constata-se que os erros de maior ocorrência estão relacionados ao posicionamento incorreto do filme, angulação vertical e horizontal incorretas. Já, a imagem do diafragma não apresentou a mesma freqüência em nosso trabalho como a encontrada por alguns autores. 
Tabela 2 - Análise dos erros de processamento

Radiografias dos diferentes anos de graduação

Curso de Odontologia - UEPG - 1996/97

\begin{tabular}{l|c|c|c|c}
\hline \multicolumn{1}{c|}{ ERROS } & $\begin{array}{c}2^{\circ} \text { ANO } \\
(\%)\end{array}$ & $\begin{array}{c}3^{\circ} \text { ANO } \\
(\%)\end{array}$ & $\begin{array}{c}4^{\circ} \text { ANO } \\
(\%)\end{array}$ & $\begin{array}{c}5^{\circ} \text { ANO } \\
(\%)\end{array}$ \\
\hline 01 - Radiografias amareladas & 13,5 & 21,0 & 15,5 & 23,0 \\
02 - Radiografias com manchas brancas & 0,0 & 1,0 & 0,0 & 0,0 \\
03 - Radiografias veladas & 1,0 & 1,0 & 4,5 & 0,0 \\
04 - Radiografias com bolhas de ar & 0,0 & 0,0 & 0,0 & 0,0 \\
05 - Reticulação do filme & 0,0 & 0,0 & 0,0 & 0,0 \\
06 - Radiografias com impressão digital & 0,0 & 1,0 & 1,0 & 1,0 \\
07 - Eletricidade estática & 0,0 & 6,0 & 0,0 & 0,0 \\
08 - Radiografias riscadas & 4,0 & 5,0 & 3,0 & 5,0 \\
\hline
\end{tabular}

O erro de processamento mais cometido foi o de radiografias amareladas, com uma média de $18,3 \%$ apontado no Gráfico 2, o que corresponde ao total de 146 radiografias; seguido do erro de radiografias riscadas, com 4,3 \% (34 radiografias); na seqüência tem-se o erro de radiografias veladas com 1,6\% (13 radiografias); eletricidade estática com 1,5\% (12 radiografias). Os demais erros de processamento analisados não tiveram freqüência significativa.

Rother e Schwarz (1976), avaliando as falhas mais freqüentes na tomada de radiografias periapicais, verificaram que $19,3 \%$ ocorrem por erros na câmara escura.

Matheus et al. (2000) constataram a presença de 13\% de radiografias amareladas. 


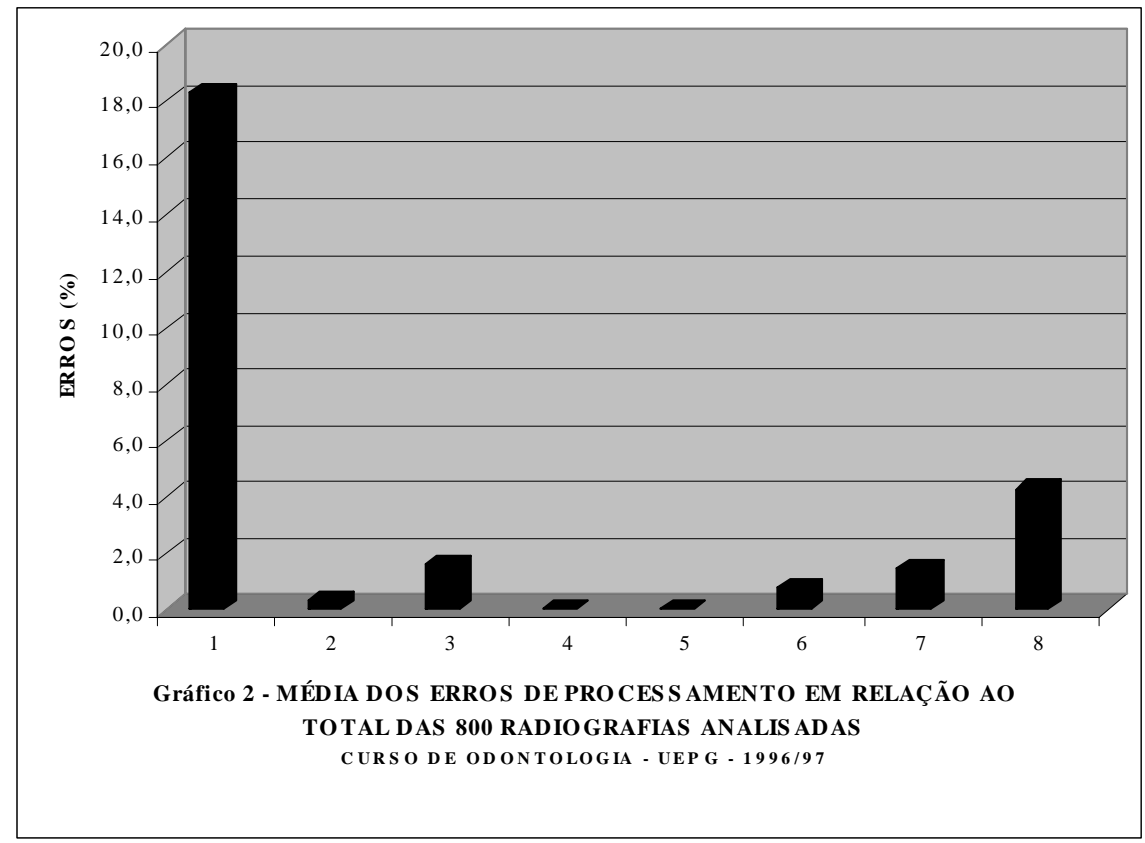

As radiografias com erros de densidade foram agrupadas numa tabela à parte, pois somente com a imagem radiográfica não há como se identificar a origem da falha, se de técnica (subexposta ou superexposta) ou de processamento (subrevelada ou superrevelada).

Tabela 3 - Análise das radiografias corretas e com erros de densidade

\begin{tabular}{l|c|c|c|c}
\hline \multicolumn{6}{c}{ Radiografias dos diferentes anos de graduação } \\
Curso de Odontologia - UEPG - 1996/97 \\
\hline \multicolumn{1}{c|}{ ERROS } & $2^{\circ}$ ANO & $3^{\circ}$ ANO & $4^{\circ}$ ANO & $5^{\circ}$ ANO \\
& $(\%)$ & $(\%)$ & $(\%)$ & $(\%)$ \\
\hline 01 - Radiografias corretas & 9,5 & 1,0 & 7,0 & 10,0 \\
02 - Radiografias claras & 40,0 & 61,0 & 63,0 & 59,0 \\
03 - Radiografias escuras & 9,0 & 1,0 & 1,0 & 3,0 \\
\hline
\end{tabular}


De acordo com a Tabela 3 e Gráfico 3, observa-se um grande número de radiografias claras com uma média de 55,8\%, totalizando 446 radiografias. O percentual de radiografias escuras foi de 3,5\% (28 radiografias).

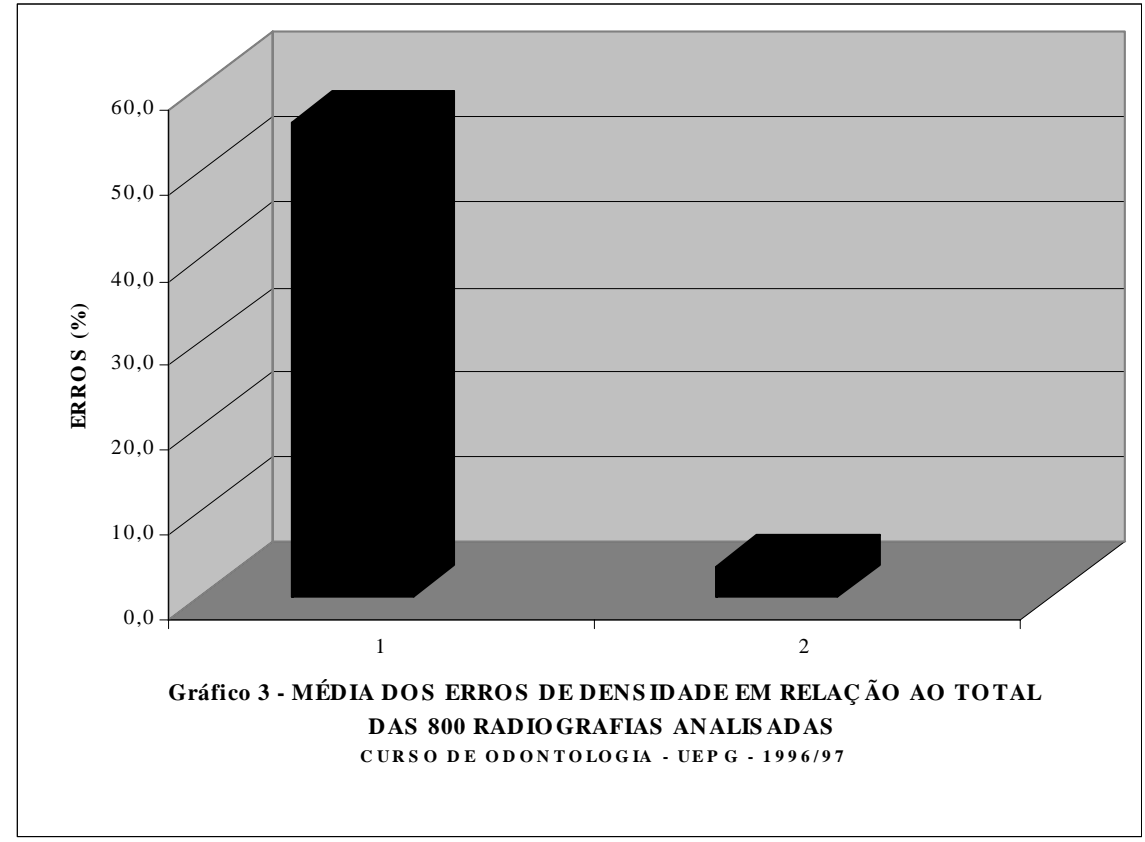

Matheus et al. (2000) realizaram um estudo semelhante na Faculdade de Odontologia da Universidade do Norte do Paraná, onde grande porcentagem dos erros estava relacionada ao processamento, neste estudo, radiografias claras foram consideradas como subprocessadas, atingindo a marca de $14,8 \%$, e as radiografias escuras, no caso superprocessadas, com $6,6 \%$. Poucos trabalhos apresentam esta classificação de erros de densidade, sendo que na maioria estes erros são classificados como de técnica ou de processamento, o que dificulta maiores comparações. No entanto, Svenson et al. (1995), consideraram esta classificação e constataram que as falhas mais comuns, em radiografias imperfeitas, estão relacionadas aos erros de angulação (27,6\%) e a densidade (22,9\%), sendo que radiografias claras foram 4 vezes mais comuns que as escuras. 
Pode-se verificar ainda, que o percentual de radiografias corretas foi baixo, com uma média de 6,9\%, o que equivale a 55 radiografias. Gasparini et al. (1992), observaram que apenas 8,5\% das radiografias analisadas por eles não apresentavam erro algum, ou seja, eram tecnicamente perfeitas e não possuíam erro de processamento.

Diante dos resultados obtidos pôde-se constatar que do total das 800 radiografias analisadas, 6,9\% apresentaram-se corretas e 93,1\% apresentaram-se incorretas, seja com erros de técnica, de processamento, de densidade ou com uma associação destes erros.

\section{Conclusões}

Dentre os erros analisados o de maior ocorrência está relacionado à Técnica: posicionamento incorreto do filme (57,0\%); seguido do erro de Densidade: radiografia clara (55,8\%). A média destes dois erros foi alta, porém não houve uma divergência muito grande entre os diferentes anos de graduação. Os demais erros analisados apresentaram um percentual de incidência baixo.

Conclui-se de um modo geral, que todos os anos de graduação apresentaram percentuais semelhantes em relação a quantidade dos erros cometidos, ocorrendo uma pequena variação no tipo de erro presente. A partir dos resultados , sugere-se que não houve uma melhora na capacidade dos alunos em relação à prática radiográfica, com a experiência adquirida no decorrer do Curso. Portanto, recomenda-se a necessidade de uma maior cobrança por parte dos professores e do próprio acadêmico, pois, radiografias com qualidade irão facilitar os procedimentos clínicos nas diversas especialidades da Odontologia, e quando arquivadas, poderão ser utilizadas no futuro sem qualquer prejuízo da imagem.

Recebido para publicação em 30/07/2002.

Aceito para publicação em 30/09/2002. 


\begin{abstract}
The radiographic exam is an important and necessary complementary tool used by professionals in health care to elaborate the diagnosis, planning and execution of the treatment, a long term follow up of clinical cases and documentation in general. In order to obtain a correct and secure interpretation, it is necessary radiographies with an acceptable pattern of quality, which will allow an adequate diagnosis. With the aim to refine the quality of the radiographic image, we analyzed intra-oral periapical radiographies, performed in 1996 and 1997 by students from the $2^{\circ}, 3^{\circ}$, $4^{\circ}$ and $5^{\circ}$ period of the Dentistry Course in UEPG, considering the following criteria of evaluation: technique, processing and radiographic density. The data obtained were compared among the different periods of graduation and the capacity of improvement, with the experience acquired during the course, was evaluated. It was ascertained that $93,1 \%$ of the radiographies showed some kind of error and the most frequents were: improper positioning of the film (57,0\%), light radiography (55,8\%), shortening of the image (24,9\%), elongation of the image (20,1\%), improper horizontal angulations $(18,4 \%)$. The authors also concluded that the index of errors showed similar percentages in all years of graduation, during the course.
\end{abstract}

Key words: intra-oral radiographies; technique/processing errors; control of quality

Endereço para contato: Eliane Maria Kreich

Rua Dr. Roberto de Jesus Portela, 287 casa 03

Ponta Grossa/PR

Tel: (42) 238-4150 - Celular: (42) 9107-3521

\title{
REFERÊNCIAS
}

1 GASPARINI, D.; SANTOMAURO VAZ, E.M.; HAITER NETO, F. et al. Análise de erros radiográficos cometidos por alunos da Faculdade de Odontologia de Piracicaba, no período de 1975 a 1988. Revista de Odontologia da USP, v. 6, n. 3/ 4, p. 107-114, jul./dez. 1992.

2 MATHEUS, R.A.; MONTEBELO FILHO, A.; TANAKA, E.E. et al. Avaliação no desempenho de acadêmicos durante tomadas radiográficas periapicais, pela técnica da bissetriz, realizadas na Faculdade de Odontologia da Universidade Norte do Paraná (Unopar). Revista da Faculdade de Odontologia de Passo Fundo, v.5, n.2, p.25-29, jul./dez. 2000. 
3 MOURSHED, F. A study of intraoral radiographic errors made by dental students. Oral Surgery, v.32, n.5, p.824-828, nov. 1971.

4 PATEL, J.R. Intraoral radiographic errors. Oral Surgery, v. 48, n. 5, p. 479-483, nov. 1979.

5 PATEL, J.R.; GREER, D.F. Evaluating student progress through error reduction in intraoral radiographic technique. Oral Surgery Oral Medicine Oral Pathology, v. 62, n. 4, p. 471-474, oct. 1986.

6 ROTHER, V.U.; SCHWARZ, H.M. Die Häufigsten Fehler bei der Anfertigung intraoraler Röntgenaufnahmen. Stomatologie Der DDR, v.26, n.12, p. 806-812, 1976.

7 SVENSON, B.; ERIKSSON, T.; KRONSTRÖM, M. et al. Quality of intraoral radiographs used for prosthodontic treatment planning by general dentists in the Public Dental Health Service. Swedish Dental Journal, v. 19, p. 47-54, 1995.

8 YACOVENCO, A. Análise dos problemas da radiografia na prática odontológica. Revista da ABRO. v. 2, n. 1, p. 29-39, jan./jun. 2001. 